\title{
Phenotypic variation in oronasal perception and the relative effects of PROP and Thermal Taster Status
}

\author{
Qian Yang ${ }^{1}$, Tracey Hollowood ${ }^{2}$, and Joanne Hort ${ }^{1}$ \\ ${ }^{1}$ Sensory Science Centre, Division of Food Sciences, University of Nottingham, Sutton \\ Bonington, Loughborough, Leicester, United Kingdom, LE12 5RD \\ ${ }^{2}$ Sensory Dimensions, Nottingham, United Kingdom, NG5 9RA
}

\begin{abstract}
Individual variation in taste perception has long been investigated, particular in relation to PROP taster status (PTS). Recently, a new marker has been identified, Thermal Taster Status (TTS), whereby individuals are categorised as thermal tasters (TTs) or thermal non-tasters (TnTs) based on their ability to perceive taste solely from temperature stimulation. The aim of this study was to investigate the incidence of thermal tasters and relative effects of PTS and TTS on oronasal sensitivity across the whole perceptual range. Both detection thresholds (ASTM E679) and intensity measures at suprathreshold level (rated on gLMS) for stimuli from a range of modalities were determined from up to 124 subjects pre-screened for their PTS and TTS. No significant differences were found within either PTS or TTS groups at detection threshold level, with one exception; TTs has a lower threshold for sucrose $(p<0.05)$. At supra-threshold level, PROP supertasters (pSTs) and medium tasters (pMTs) rated stimuli higher than non-tasters, and a consistent trend was observed that TTs rated stimuli higher than TnTs, although only ratings for temperature (warm and cold) reached significance. Global analyses applied across each modality, showed that in general TTs rated gustatory and trigeminal modalities significantly higher than TnTs, whilst this was not the case for olfactory stimuli, indicating that the mechanism for increased perception for TTs may be located in the oral cavity. PTS and TTS were shown to be independent phenotypes, but interestingly, ANOVA revealed significant interactions between TTS and PTS across the three modalities. Most notably, within pMTs, TTs rated stimuli intensity higher than TnTs, while the opposite trend was observed for pSTs. The intensity advantage gained by thermal tasters appears to be more apparent for pMTs than the already highly sensitive pSTs.
\end{abstract}

Keywords: Thermal taster status, PROP taster status, Detection threshold, Suprathreshold, Temperature, Oronasal sensitivity 


\section{Introduction}

Oronasal sensitivity varies greatly among individuals and is purported to affect food consumption behaviour and subsequently a range of health and disease outcomes (Stewart, et al., 2010, Villarino, et al., 2009, Ullrich, et al., 2004). Many factors contribute to this individual variation, such as age, gender, ethnic origin and different phenotypes (Mojet, et al., 2003, Mojet, Leshem, et al., 2003, Hirokawa, et al., 2006, Pickering, et al., 2010, Lim, et al., 2008). Individual difference in perceiving 6-npropylthiouracil (PROP) bitterness is the most studied source of individual variation in taste perception since Blakeslee and Fox first discovered it over eight decades ago (Blakeslee, et al., 1932). The gene contributing to PROP perception has since been identified as TAS2R38 (Kim, et al., 2003), a member of the bitter taste receptor family, which has two common molecular forms (PAV and AVI). Individuals with two PAV alleles are very sensitive to PROP (Supertasters (pSTs)), those with two AVI alleles are insensitive to PROP (non-tasters (pNTs)) and those with one of each are moderately sensitive (medium tasters (pMTs)) (Duffy, et al., 2004). Previous studies have also linked sensitivity to PROP with increased taste, trigeminal and olfactory perceptions (Bartoshuk, 1979, Bajec \& Picking, 2008, Tepper \& Nurse 1997, Prescott \& Swain-Campbell, 2000, Yackinous \& Guinard, 2001, Pickering, et al., 2006, Gent \& Bartoshuk, 1983, Bartoshuk, et al., 1998). A greater density of fungiform papillae is associated with pSTs compared to medium and non-tasters (Miller, et al., 1990, Bartoshuk, et al., 1994, Delwiche, et al., 2001, Essick, et al., 2003), and thus pSTs have more chorda tympani and trigeminal nerve fibers. This is likely to explain the advantage of pSTs over the other two groups in terms of increased taste (Gent \& Bartoshuk 1983, Lim, et al., 2008, Bajec \& Pickering, 2008), and trigeminal sensitivity (Prescott \& Swain-Campbell, 2000, Tepper \& Nurse, 1998). Surprisingly, pSTs have also been shown to demonstrate increased perception to olfactory stimuli (Pickering, et al., 2006) and increased negative emotions when viewing film clips (Macht \& Mueller, 2007). Such findings have suggested that mechanisms at a higher level in the CNS system may contribute to increased sensitivity. However, sensitivity to PROP has not always found an association with increased responsiveness to other oronasal sensory stimuli (Ly \& Drewnowski, 2001, Yackinous \& Guinard, 2001); and as such other unknown factors are likely to contribute to individual variation in oronasal perception.

In 2000, Cruz and Green found a new marker of individual variation in oral sensation: thermal taste. Individuals who had the ability to perceive 'phantom' taste sensations during heating or cooling of a small area of tongue were described as 'thermal tasters' (TTs). Thermal sweetness was most often tasted on the tip of the tongue when it was re-warmed from an initial cooling period $\left(20^{\circ} \mathrm{C}\right.$ to $\left.35^{\circ} \mathrm{C}\right)$, whereas cooling the tongue tip (below $10^{\circ} \mathrm{C}$ ) evoked a sour or salty taste sensation in some individuals (Cruz \& Green, 2000). There is some suggestion that this phenomenon may be linked to the TRPM5 cation channel, which is a highly temperature-sensitive, heat activated channel, with a key role in the transduction of umami, sweet and bitter taste (Talavera, et al., 2005). It has been further suggested that other tastes, such as salty and sour, perceived by TTs may be linked to the temperature sensitivity of the channels involved in their chemical transduction (Talavera, et al., 2007). Interestingly, TTs do not only have the ability to perceive 'phantom taste' from temperature stimulation, but have also been reported to have a heightened response to taste and some trigeminal stimuli compared to Thermal non-tasters (TnTs) (Green, et al., 2005, Green \& George, 2004, Bajec \& Pickering, 2008). Green and 
George and Green (2004) also reported that TTs rated an aroma (vanilla), both retronasally and orthonasally sensed as more intense than TnTs, suggesting that a higher 'central gain' mechanism may also be involved in the increased sensory perception.

Both PROP taster status and thermal taster status appear to play a role in oronasal sensitivity at supra-threshold level. However, to date little research looking at detection level sensitivity has been reported, especially in relation to TTS. The present study examines the impact of PTS and TTS on both detection and suprathreshold sensitivities across a range of gustatory, trigeminal and olfactory modalities. In addition the relationship between and relative effects of these two phenotypes is investigated.

\section{Material \& Methods}

\subsection{Subjects}

204 volunteers (132 female, 72 male mean age 42 range 16-75 years) were recruited from the Sensory Dimensions Ltd (Nottingham, UK) consumer database, and students from the University of Nottingham. All subjects signed to say they had given informed consent and were given an incentive for participating. Of the 204 subjects, 124 subjects attended the detection threshold study and 112 subjects were invited back to attend the supra-threshold study.

\section{2. gLMS Scale Training}

Prior to phenotype screening, all subjects were trained in the use of the gLMS scale to measure the intensity of temperature and any subsequence taste sensations perceived during all trials. To familiarise subjects with the gLMS scale and facilitate its correct use, subjects were given a gLMS scale reference sheet. They were asked to write down the strongest sensation of any kind they had experienced previously or the strongest sensation they could imagine happen to them, which represented the top of the scale. They were then asked to rate the intensities of 15 remembered sensations (Bartoshuk, et al., 2002), relative to their own strongest sensation. Their reference sheet was always presented alongside all scales in subsequent experiments.

\subsection{Thermal Taster Status Determination}

In order to heat or cool a small area of the tongue, a circular intra-oral ATS (advanced thermal stimulator) peltier thermode with a truncated cone area of 28.26 $\mathrm{mm}^{2}$ at the top (Medoc, Israel) was used. For hygiene purposes, the thermode was wiped with $99 \%$ ethanol (Fisher Scientific, UK) between subjects and covered with a fresh piece of tasteless plastic wrap (Tesco, UK) for each subject. Subjects were asked to extend their tongue, and gently place the thermode on the anterior tip of the tongue with the guidance of researcher. Subjects were instructed to hold the thermode firmly in place during all temperature trials.

Before testing, subjects were presented with their own reference sheet, and two further gLMS scales for each temperature trial. One was labeled 'temperature' and subjects were asked to rate the maximum intensity of the temperature during the trial. The second scale was provided to record the quality and intensity of any taste 
sensations perceived during temperature stimulation. Taste options listed below this scale, were 'no sensation', 'sweet', 'bitter', 'sour', 'salty', 'savoury', 'metallic' and 'other, please specify'. Subjects were instructed to tick the taste options and rate the intensity of the taste sensation at its maximum intensity if they had perceived one. Two temperature trials were used: a warming trial and a cooling trial. For each subject two repetitions of each trial were obtained. Before each temperature trial, a baseline trial was applied at body temperature $\left(37^{\circ} \mathrm{C}\right)$ and held for $10 \mathrm{~s}$. For the warming trial the probe started at $35^{\circ} \mathrm{C}$, was then cooled to $15^{\circ} \mathrm{C}$ and then rewarmed to $40^{\circ} \mathrm{C}$ and held for $1 \mathrm{~s}$. For the cooling trial the probe started at $35^{\circ} \mathrm{C}$ and was cooled to $5^{\circ} \mathrm{C}$ and held for $10 \mathrm{~s}$. The temperature ramp for all trials was $1^{\circ} \mathrm{C} / \mathrm{s}$. Warming trials preceded cooling trails to avoid possible adaptation from the intense, sustained cold stimulation. A break of two minutes was given before proceeding to the next trial to allow the tongue temperature/sensation to return to normal.

\subsection{PROP Taster Status Determination}

A $0.32 \mathrm{mM}$ solution of PROP (Sigma Aldrich, UK) solution was prepared by dissolving PROP in water on a low heat stirring plate. Each subject was instructed to roll a saturated cotton bud, which had previously been dipped in the PROP solution $\left(22^{\circ} \mathrm{C} \pm 2\right)$, across the tip of the tongue for approximately $3 \mathrm{~s}$. They then rated the intensity of the solution at its maximum using the gLMS scale. After a 5 minute break, the procedure was repeated to collect duplicate ratings.

\subsection{PROP and Thermal Taster Status Categorisation}

Thermal tasters (TTs) were defined as those individuals reporting taste sensations during both warming and cooling trials rated above 'weak' on the gLMS. Thermal non-tasters (TnTs) were defined as those who did not perceive any taste sensation in any of the temperature trials. Notably, this left a group of people uncategorised (Uncat) due to inconsistencies in reporting taste sensations throughout the trials.

PROP taster status was defined based on mean PROP intensity ratings: PROP nontasters (pNTs) were defined as those rating below 'barely detectable'; PROP medium tasters (pMTs) were those rating above barely detectable but below moderate; and PROP super-tasters (pSTs) were those rating above 'moderate' on the gLMS (Lim, et al., 2008).

\subsection{Detection Threshold Measurement}

All samples were freshly prepared with Evian water (Danone, France) on the morning of the testing day. Seven stimuli were tested - sucrose $(1.8 \mathrm{mM}-20.4 \mathrm{mM}$, Silverspoon, UK) sodium chloride (1.3mM-34.2mM), caffeine $(0.15 \mathrm{mM}-2.32 \mathrm{mM}), \mathrm{N}-$ Ethyl-2-isopropyl-5-methylcyclohexanecarboxamide (WS3) $\quad(0.43 \mu \mathrm{M}-47.3 \mu \mathrm{M})$, capsaicin (3.3nM-556nM), ethyl butyrate $(8.6 \mathrm{nM}-3443 \mathrm{nM})$ and isoamyl acetate (7.7nM-3070nM) (all Sigma Aldrich, UK). ASTM standard E679 was employed (International ASTM, 2004) to determine individual detection thresholds. In order to obtain a testing series of nine 3AFC presentations, a constant dilution factor (step factor) was used for each stimulus. The concentration series for each stimulus was initially established from previous literature (Devos, et al., 1990, BS-5929-7, 1992, Toontom, et al., 2001), and then modified by a pilot test in our laboratory. 
Subjects attended four 1-hour sessions. Each threshold test comprised nine 3-AFC tests, presented in ascending order. Subjects completed the whole set of nine 3-AFC tests for each stimulus using Compusense (Compusense Five 5.4, Canada). Stopping rule 3 (Peng, et al., 2012) was employed to calculate each individual's best-estimate threshold (BET), which was taken as the geometric mean of the concentration at the last miss and the next higher concentration when 3 correct choices occurred in a row. If subjects missed at the highest concentration an assumption was made that the subject would answer correctly at the next concentration level, and hence the BET was the geometric mean of the last concentration and the predicted next concentration (last concentration * step factor) (International ASTM, 2004).

\subsection{Supra-threshold Measurement}

All samples were prepared with Evian water the day prior to testing, stored in the fridge at $4^{\circ} \mathrm{C}$, and brought to room temperature $\left(22 \pm 2^{\circ} \mathrm{C}\right)$ in advance of testing. The stimuli concentrations were $0.1 \mathrm{M}$ and $1 \mathrm{M}$ sucrose; $0.056 \mathrm{M}$ and $0.56 \mathrm{M} \mathrm{NaCl} ; 5.6 \mathrm{mM}$ and $56 \mathrm{mM}$ citric acid (Sigma, UK); $1.8 \mathrm{mM}$ and $56 \mathrm{mM}$ caffeine; $1.8 \mu \mathrm{M}$ and $32 \mu \mathrm{M}$ Capsaicin (Green, et al., 2005, Green \& George, 2004). Capsaicin was first dissolved in ethanol (Fisher Scientific, UK), and then diluted with water. Samples were applied to the anterior tip of tongue using cotton buds that had been previously dipped in each test solution. At least 1 minute break was given between each stimulus, although a longer break was allowed if subjects could still perceive the previous stimulus. All subjects repeated gLMS scale training before performing the supra-threshold sensitivity measurement. They were encouraged to refer back to their own reference sheet and rate the intensity of each stimulus on the computerised gLMS (Compusense Five 5.4, Canada).

\subsection{Data Analysis}

The percentage of individuals classified according to each phenotype during screening was ascertained. In addition, the frequencies of the taste sensations perceived by TTs during the warming and cooling trials were determined.

Each individual BET was log transformed before further statistical analysis. Perceived intensity ratings from the supra-threshold tests were also log transformed as gLMS data is typically log-distributed. Prior to log transformation all zeroes were replaced with 0.5 .

One-way Analysis of Variance (ANOVA) was applied to the combined TTs and TnTs data (i.e. unclassified individuals were removed from the data set) to determine if TTS significantly affected detection thresholds for each individual stimulus. One-way ANOVA was also applied to determine if significant differences in detection threshold existed between different PTS groups for each stimulus. Furthermore, a two-way ANOVA was applied to determine if interactions occurred across TTS and PTS for detection thresholds.

To determine if TTS had a significant effect at suprathreshold level, a one-way ANOVA was applied to the combined TTs and TnTs data for each individual stimulus, but also on the global data and data pooled for each modality (taste, aroma, trigeminal). One-way ANOVA, with post hoc Tukeys multiple comparison tests where appropriate, were used to determine if PTS had a significant effect on 
intensity perception of each individual stimulus. A Chi Square analysis was applied to determine if any relationship existed between TTS and PTS classification. Pearson's correlation coefficient was calculated to examine the relationship between detection threshold and supra-threshold sensitivities. All analyses were performed using SPSS, version 21 (SPSS IBM, USA).

An $\alpha$-risk of 0.05 was set for all statistical analyses.

\section{Results}

\subsection{Incidence of TTS and Tastes Perceived}

Following the strict classification criteria used in this study, of the 204 subjects 56 subjects were classified as TTs, and 61 subjects were classified as TnTs, the remaining 87 subjects were uncategorised (Uncat). There was an equal distribution of genders across all classifications indicating no relationship between gender and TTS.

The most frequent taste sensations reported by TTs during both temperature trials were metallic, sweet, bitter and sour. Other tastes were occasionally reported including salty, savoury, spicy and tingling (fig.1). It is interesting to note that sweetness was reported $14 \%$ more frequently during the warming trial than cooling, and that both bitter and sour were reported 7 to $8 \%$ more frequently in the cooling trial. The average log perceived intensity of the taste sensations were $1.25 \pm 0.35$ for warming trial and $1.45 \pm 0.29$ for cooling trial, which equates to around moderate for the warming trial and just below strong for the cooling trial on the gLMS scale.

\subsection{Oronasal Sensitivity at Detection Threshold Level}

Between 37 to 41 TTs and 32 to 39 TnTs participated in the detection threshold tests. (The numbers vary as not all subjects were able to attend all sessions.) Oneway ANOVA revealed that TTs had a significantly lower threshold for sucrose $(p=0.032)$. No significant difference was observed for any other oronasal stimuli at detection threshold (fig. 2).

Between 24-31 pSTs, 44-55 pMTs and 18-22 pSTs participated in the detection thresholds tests (Numbers vary as not all subjects were able to attend all sessions.) No significant difference ( $p>0.05)$ was observed for any detection threshold among PTS groups (fig.3).

Two-way ANOVA indicated no significant TTS*PTS interaction for any oronasal stimuli at detection threshold level.

\subsection{Oronasal Sensitivity at Supra-threshold Level}

\subsubsection{Thermal Taster Status}

One-way ANOVA was performed on ratings of each individual stimulus in order to compare TTs and TnTs. The results showed that TTs rated both warm and cold stimuli significantly higher than TnTs $(p<0.05)$. Data for salt (high), caffeine (low) also approached significance $(p<0.1)$. No significant difference was observed for any other oronasal stimuli among TTS groups. However, a trend was observed that TTS rated intensities higher than TnTs for most of the stimuli (fig. 4). Consequently, a 
further analysis was conducted by performing one-way ANOVA on global intensity ratings, and data grouped by modality. The results showed that overall TTs rated stimuli intensities significantly higher than TnTs $(p<0.05)$. On the whole, TTs rated both taste and trigeminal modality intensities significantly higher $(p<0.05)$ than TnTs, however this was not the case for aroma.

\subsubsection{PROP Taster Status.}

One-way ANOVA, followed by Tukey's HSD tests, was performed on each individual stimulus among PTS groups with results summarised in fig. 5 . The results show that pSTs rated all stimuli as significantly more intense than pNTs $(p<0.05)$, except for EB ortho (low and high). Several additional samples were also significantly more intense for pMTs than pNTs (sucrose (low and high), salt (low and high), caffeine (low), PROP, EB retro (high) and EB ortho (low)). No significant differences $(p>0.05)$ in stimulus ratings were found between pSTs and pMTs with the exception of PROP.

\subsubsection{Relative Effects of PTS and TTS}

The two thermal taster groups were similarly, although not completely balanced for PTS. Of the 35 TTs, 11 were pSTs, 20 were pMTs and 4 were pNTs. Of the 37 TnTs, 9 were pSTs, 20 were pMTs, 8 were pNTs. The Chi-square test applied to examine the relationship between TTS and PTS classification revealed that the two phenotypes were independent of each other $(p>0.05)$, as has been previously suggested (Bajec \& Pickering, 2008).

Interestingly, two-way ANOVA, performed on global intensity ratings, revealed a significant interaction between TTS and PTS. Further two-way ANOVA on pooled data for each modality revealed significant interactions for taste $(p<0.05)$ and interactions approached significance for both aroma and trigeminal modalities $(p<0.1)$ (see interaction plots in fig. 6). Observations of these plots revealed an interesting trend that pSTs who were TTs rated the intensity of all three modalities lower than pSTs who were TnTs, while the opposite trend was clearly observed for pMTs i.e. the rating of pMTS who were TTs were higher than pMTs who were TnTs. No clear trend was observed in pNT group.

Although, no significant interactions were found between TTS and PTS when analyzing the individual attributes the same trend was observed and approached significance for sucrose ( $p=0.083$ ) (fig. 7 ).

\subsubsection{Further considerations}

The above observations prompted a more in-depth analysis looking at TTS effects within each separate PTS group. Within the pSTs, no significant differences in intensity measures between TTs and TnTs were found for any oronasal stimuli, although a trend that TTs rated most oral stimuli lower than TnTs was observed (fig. 8a). In pMTs, the trend was the opposite such that TTs rated stimulus intensities higher than TnTs, with the exception of PROP. Indeed, the ratings of sucrose (low), caffeine (low), warming and cooling were found to be significantly more intense for TTs $(p<0.05)$, and sucrose (high) approached significance $(p<0.1)$, (fig. 8b).

\section{Discussion}

\subsection{Thermal Stimulation of Taste}


Cruz and Green (2000) revealed that about $50 \%$ of their test population were TTs, while Bajec and Pickering (2008) claimed a lower prevalence of TTs at $20 \%$ with, $40 \%$ of the test population identified as TnTs. In this current study, results indicated that about $27 \%$ of the subjects were TTs and $28 \%$ were TnTs. The differences reported between studies are probably due to categorisation methods. Pickering's group used much stricter criteria, only classifying subjects as TTs if they consistently reported taste sensations through all temperature trails, and reported the same taste sensation within replicates. Green's group however, classified subjects as TTs if they reported taste sensations in either warming or cooling trials for both replicates. The criteria in this current study are similar to Pickering's, but did not require the same taste sensation to be reported across replicates. In any case, it is clear that TTs comprise a large proportion of the population and warrant further investigation.

The present study showed that both heating and cooling most commonly evoked a metallic taste. Metallic taste has also been reported by TTs in other studies, but not as frequently (Bajec, et al., 2012). Previous research has suggested two likely mechanisms for metallic sensation: i) that it is a true gustatory mechanism evoked by electrical stimulation of taste receptors in fungiform papillae and not affected by nasal occlusion (Lawless, et al., 2005); ii) and/or that it may be multimodal involving gustatory, olfactory and trigeminal pathways (Epke, et al., 2009), particularly as metallic sensation from ferrous sulphate has been shown to be modified by nasal occlusion (Lawless, et al., 2004). Interestingly, in the present study, temperature stimulation was applied on the anterior tip of the tongue, where fungiform papillae are housed. This raises the possibility that temperature stimulation may activate a response similar to that of an electrical current, through stimulation of gustatory pathways or trigeminal nerves resulting in perception of metallic, and potentially, other tastes. It is also possible that the increased reporting of metallic sensation in this study was due to the listing of the metallic descriptor as one of the taste options whilst previous studies required subjects to proffer descriptors themselves or had no specific metallic option (Lawless, et al., 2005).

Beyond metallic taste, this study agrees with previous research that sweet taste is reported more often during the warming trial, and that bitter and sour are reported more often during the cooling trial (Cruz \& Green, 2000). Evidence that increasing temperature from $15^{\circ} \mathrm{C}$ to $35^{\circ} \mathrm{C}$ markedly enhanced gustatory nerve response to sweet compounds in wild-type mice, whilst residual gustatory responses in TRPM5 knockout mice did not change with temperature, implies that 'thermal sweetness' could be evoked by temperature activation of the TRPM5 channel modulation. TRPM5 is a highly temperature sensitive, heat activated channel, which has a key role in the perception of sweet, umami and bitter taste (Talavera, et al., 2005, Talavera, et al., 2007). However, studies show that metallic taste is unlikely to be activated by TRPM5 (Riera, et al., 2009), indicating that TRPM5 may not be the only potential mechanism for 'phantom taste' and that further research is needed to determine the mechanisms involved.

\subsection{Oronasal Sensitivity}

No difference was found in detection threshold for any compound investigated between TTS groups with the exception of sucrose. TTs had significantly lower sucrose thresholds than TnTs again providing further evidence that individual variation in the TRPM5 channel may play a role in observed TTS behaviours. 
The Pearson correlation test revealed no significant correlation $(p>0.05)$ between detection threshold and supra-threshold sensitivity (data not shown), a disassociation echoed by previous research into lingual tactile (Fucci, et al., 1985), PROP (Bartoshuk, et al., 1994) and caffeine sensitivity (Keast, et al., 2007). Indeed, perceptual mechanisms operating at detection threshold and supra-threshold levels are likely to operate differently as there may be multiple taste transduction mechanisms that are activated at different concentrations (Keast, et al., 2007). Detection simply involves determining if a stimulus is there or not, whereas with perception at supra-threshold levels there is a need to determine the level of intensity which involves higher central gain cortical activity (Fucci, et al., 1985). In agreement with previous studies (Green \& George, 2004, Bajec \& Pickering, 2008), a global trend was observed that TTs have a heightened response over TnTs to supra-threshold stimuli. The fact that this difference was not significant for individual attribute intensity ratings, apart from temperature could be due to lack of power, as in this study, replicate data were not obtained. In addition, although training on using the gLMS scale was given, the narrow range covered by the stimuli (between weak ' 6 ' and very strong ' 54 ') on this absolute scale (0-100) may make subtle differences in intensity more difficult to determine. Currently however, no other scale is available to facilitate comparison of absolute differences in intensity.

A recent $\mathrm{fMRI}$ study showed that cortical response to a carbonated sweet aqueous stimulus was significantly increased in several areas of the brain including the somatosensory cortex of thermal tasters (Clark, 2011). The observation in the current study that TTs gave globally significantly higher ratings at a supra-threshold level was mirrored for both taste and trigeminal modalities and was a trend maintained for each individual attribute stimulus. Clark (2011) hypothesised that cross wiring between the taste and trigeminal nerves in thermal tasters could cause the phantom taste responses stimulated by the trigeminal stimulus (temperature) and could similarly allow both nerves to be activated to a taste stimulus, increasing intensity response and cortical activation (Clark, 2011). In the current study, no difference in aroma intensity ratings of ethyl butyrate was observed across TTS. This further supports the hypothesis, as the co-innervation of taste and trigeminal receptors would have limited effect on aroma perception.

\subsection{Relative Effect of PROP and Thermal Taster Status}

The data in this study confirmed that TTS and PTS phenotypes are independent, adding to the evidence from Bajec and Pickering 2008 that TTS and PTS operate via different mechanisms. However, interactions identified in this study indicate that their relative effects may have significant impact on perception for certain phenotypic combinations.

A pattern was observed that TTs had, if anything a slightly weakened response to oronasal stimuli in pST group, but a heightened response in the PMT group, no clear effect was seen in pNT group. It could be hypothesized that these observations may be linked to the differing number of papillae closely linked with PTS (Miller \& Reedy, 1990).

High numbers of fungiform papillae housing taste and trigeminal receptors are associated with pSTs and hence their high sensitivity to oral stimuli. The data in this study suggests that any advantage gained by also being a TT does not impact on 
the perception of these already supersensitive individuals. However, for pMTs, who have moderate numbers of papillae, being a TT appears to impact on perceived intensity such that there is a considerable gain in perception compared to their TnT counterparts. For pNTs who tend to have very few fungiform papillae, the enhanced impact of TTs on oronasal sensitivity could be restricted as they have will have less gustatory and trigeminal nerve endings. This is a hypothesis and further studies are clearly needed to understand the mechanism behind the relative effects of these different phenotypes on perception.

\section{Conclusions}

This study continues to highlight the considerable incidence of thermal taster's in the population and the range of phantom tastes they perceive. The high incidence of phantom metallic taste is interesting and warrants further investigation particularly if this normally unwanted sensation is evoked in TTS when consuming products which rapidly cool or heat the tongue.

This study also revealed that the increased intensity perceived by these phenotypes at supra-threshold does not mean that they have lower detection thresholds, at least for the range of attributes tested here, and suggests different mechanisms operate at detection and supra-threshold levels.

Not surprisingly the data here confirmed that pSTs have a higher sensitivity to oral stimuli, and a trend was also observed, which was significant across taste and trigeminal modalities, that TTs have an increased perception of oral stimuli. As no differences in perception of aroma stimuli were observed it seems more likely that the mechanism behind increased perception in TTS is at the periphery but that several mechanisms may be involved. Of considerable interest were the findings relating to the relative impact of the two independent phenotypes such that it is the perception of pMTs that seems most effected by TTS. More research is required to fully understand the reasons why, but number of fungiform papillae is one area that may be implicated.

\section{References}

Bajec, M. R., \& Pickering, G. J. (2008). Thermal taste, PROP responsiveness and perception of oral sensations. Physiology and Behavior, 95, 581-590.

Bajec, M. R., Pickering, G. J., \& Decourville, N. (2012). Influence of stimulus temperature on orosensory perception and variation with taste phenotype. Chemosensory Perception, 5, 243265.

Bartoshuk, L. M. (1979). Bitter taste of saccharin related to the genetic ability to taste the bitter substance 6-Propylthiouracil. Science, 205, 934-935.

Bartoshuk, L. M., Duffy, V. B., Fast, K., Green, B. G., Prutkin, J., \& Snyder, D. J. (2002). Labeled scales (e.g., category, Likert, VAS) and invalid across-groupcomparisons: what we have learned from genetic variation in taste. Food Quality and Preference, 14, 125-138.

Bartoshuk, L. M., Duffy, V. B., Lucchina, L. A., Prutkin, J., \& Fast, K. (1998). PROP (6-npropylthiouracil) supertasters and the saltiness of $\mathrm{NaCl}$. Olfaction and Taste Xii, 855, 793-796.

Bartoshuk, L. M., Duffy, V. B., \& Miller, I. J. (1994). Ptc/Prop Tasting - anatomy, psychophysics, and sex effects. Physiology and Behavior, 56, 1165-1171.

Blakeslee, A. F., \& Fox, A. L. (1932). Our different taste worlds - P T C as a demonstration of genetic differences in taste. Journal of Heredity, 23, 97-107.

Cerf-Ducastel, B., Haase, L., \& Murphy, C. (2012). Effect of magnitude estimation of pleasantness and intensity on fMRI activation to taste. Chemosensory Perception, 5, 100-109. 
Clark, R. A., (2011). Multimodal flavor perception: The impact of sweetness, bitterness, alcohol content and carbonation level on flavor perception. Unpublished PhD thesis. University of Nottingham.

Cruz, A., \& Green, B. G. (2000). Thermal stimulation of taste. Nature, 403, 889-892

Delwiche, J. F., Buletic, Z., \& Breslin, P. A. S. (2001). Relationship of papillae number to bitter intensity of quinine and PROP within and between individuals. Physiology and Behavior, 74, 329-337.

Devos, M., Patte, F., Rouault, J., Laffort, P., \& Van Gemert, L.J.(1990). Standardized human olfactory thresholds. New York: Oxford University Press.

Duffy, V. B., Davidson, A. C., Kidd, J. R., Kidd, K. K., Speed, W. C., Pakstis, A. J., Reed, D. R., Snyder, D. J., \& Bartoshuk, L. M. (2004). Bitter receptor gene (TAS2R38), 6-n-propylthiouracil (PROP) bitterness and alcohol intake. Alcoholism, Clinical and Experimental Research, 28, 1629-1637.

Epke, E. M., McClure, S. T., \& Lawless, H. T. (2009). Effects of nasal occlusion and oral contact on perception of metallic taste from metal salts. Food Quality and Preference, 20, 133-137.

Essick, G. K., Chopra, A., Guest, S., \& McGlone, F. (2003). Lingual tactile acuity, taste perception, and the density and diameter of fungiform papillae in female subjects. Physiology Behavior, 80, 289-302.

Fucci, D., Harris, D., \& Petrosino, L. (1985) Threshold and suprathreshold correlations for the oral tactile sensory mechanism. Journal of speech and hearing research, 28, 331-335.

Galindo-Cuspinera, V., Waeber, T., Antille, N., Hartmann, C., Stead, N., \& Martin, N. (2009). Reliability of threshold and suprathreshold methods for taste phenotyping: characterization with PROP and sodium chloride. Chemosensory Perception, 2, 214-228.

Gent, J. F., \& Bartoshuk, L. M. (1983). Sweetness of sucrose, neohesperidin dihydrochalcone, and saccharin is related to genetic ability to taste the bitter substance 6-n-propylthiouracil. Chemical Senses, 7, 265-272.

Green, B. G., Alvarez-Reeves, M., George, P., \& Akirav, C. (2005). Chemesthesis and taste: Evidence of independent processing of sensation intensity. Physiology and Behavior, 86, 526537.

Green, B. G., \& George, P. (2004). 'Thermal taste' predicts higher responsiveness to chemical taste and flavor. Chemical Senses, 29, 617-628.

Hirokawa, K., Yamazawa, K., \& Shimizu, H. (2006). An examination of sex and masculinity/femininity as related to the taste sensitivity of Japanese students. Sex Roles, 55, 429-433.

International ASTM (2004). Standard practice E679-04. Standard practice for determination of odor and taste thresholds by a forced-choice ascending concentration series method of limits. Philadelphia, PA, American Society for Testing and Materials.

Keast, R. S., \& Roper, J. (2007). A complex relationship among chemical concentration, detection threshold, and suprathreshold intensity of bitter compounds. Chemical Senses, 32, 245-53.

Kim, U. K., Jorgenson, E., Coon, H., Leppert, M., Risch, N., \& Drayna, D. (2003). Positional cloning of the human quantitative trait locus underlying taste sensitivity to phenylthiocarbamide. Science 299, 1221-1225.

Lawless, H. T. (2010). A simple alternative analysis for threshold data determined by ascending forced-choice methods of limits. Journal of Sensory Studies, 25, 332-346.

Lawless, H. T., Schlake, S., Smythe, J., Lim, J. Y., Yang, H. D., Chapman, K., \& Bolton, B. (2004). Metallic taste and retronasal smell. Chemical Senses, 29, 25-33.

Lawless, H. T., Stevens, D. A., Chapman, K. W., \& Kurtz, A. (2005). Metallic taste from electrical and chemical stimulation. Chemical Senses, 30, 185-194.

Leshem, M., Katz-Levin, T., \& Schulkin, J. (2003). Calcium taste preference and sensitivity in humans: I. Gender comparisons. Physiology and Behavior, 78, 403-407.

Lim, J. Y., Urban, L., \& Green, B. G. (2008). Measures of individual differences in taste and creaminess perception. Chemical Senses, 33, 493-501.

Ly, A., \& Drewnowski, A. (2001). PROP (6-n-propylthiouracil) tasting and sensory responses to caffeine, sucrose, neohesperidin dihydrochalcone and chocolate. Chemical Senses, 26, 41-47.

Macht, M., \& Mueller, J. (2007). Increased negative emotional responses in PROP supertasters. Physiology and Behavior, 90, 466-472.

Miller, I. J., \& Reedy, F. E. (1990). Variations in human taste bud density and taste intensity perception. Physiology and Behavior, 47, 1213-1219.

Mojet, J., Heidema, J., \& Christ-Hazelhof, E. (2003). Taste perception with age: Generic or specific losses in supra-threshold intensities of five taste qualities? Chemical Senses, 28, 397-413. 
Peng, M., Jaeger, S. R., \& Hautus, M. J. (2012). Determining odour detection thresholds: Incorporating a method-independent definition into the implementation of ASTM E679. Food Quality and Preference, 25, 95-104.

Pickering, G. J., Haverstock, G., \& DiBattista, D. (2006). Evidence that sensitivity to 6-npropylthiouracil (PROP) affects perception of retro-nasal aroma intensity. Agriculture and Environment, 4, 15-22.

Pickering, G. J., Moyes, A., Bajec, M. R., \& Decourville, N. (2010). Thermal taster status associates with oral sensations elicited by wine. Australian Journal of Grape and Wine Research, 16, 361367.

Prescott, J., \& Swain-Campbell, N. (2000). Responses to repeated oral irritation by capsaicin, cinnamaldehyde and ethanol in PROP tasters and non-tasters. Chemical Sense, 25, 239-246.

Riera, C. E., Vogel, H., Simon, S. A., Damak, S., \& Coutre, J. (2009). Sensory attributes of complex tasting divalent salts are mediated by TRPM5 and TRPV1 channels. The Journal of Neuroscience, 29, 2654-2662.

British Standard Method for Sensory Analysis of Food. Part 7: Investigating sensitivity of taste. BS 5929-7: 1992, ISO 3972:1991.

Stewart, J. E., Feinle-Bisset, C., Golding, M., Delahunty, C., Clifton, P. M., \& Keast, R. S. J. (2010). Oral sensitivity to fatty acids, food consumption and BMI in human subjects. British Journal of Nutrition, 104, 145-152.

Talavera, K., Ninomiya, Y., Winkel, C., Voets, T., \& Nilius, B. (2007). Influence of temperature on taste perception. Cellular Molecular Life Science, 64, 377-381.

Talavera, K., Yasumatsu, K., Voets, T., Droogmans, G., Shigemura, N., Ninomiya, Y., Margolskee, R. F., \& Nilius, B. (2005). Heat activation of TRPM5 underlies thermal sensitivity of sweet taste. Nature, 438, 1022-1025.

Tepper, B. J., \& Nurse, R. J. (1998). PROP taster status is related to fat perception and preference. Olfaction and Taste Xii, 855, 802-804.

Tepper, B. J., \& Nurse, R. J. (1997). Fat perception is related to PROP taster status. Physiology and Behavior, 61, 949-954.

Toontom, N., Meenune, M., \& Posri, W. (2001). Detection threhsold perceived by light, moderate and heavy chili users. The 12th Asean food conference 2011, 590-594.

Ullrich, N. V., Touger-Decker, R., O'Sullivan-Maillet, J., \& Tepper, B. J. (2004). PROP taster status and self-perceived food adventurousness influence food preferences. Journal of the American Dietetic Association, 104, 543-549.

Villarino, B. J., Fernandez, C. P., Alday, J. C., \& Cubelo, C. G. R. (2009). Relationship of prop (6-NPropylthiouracil) taster status with the body mass Index and food preferences of Filipino adults. Journal of Sensory Studies, 24, 354-371.

Whitehead, M. C., Beeman, C. S., \& Kinsella, B. A. (1985). Distribution of Taste and General Sensory Nerve-Endings in Fungiform Papillae of the Hamster. American Journal of Anatomy, 173, 185201.

Yackinous, C., \& Guinard, J. X. (2001). Relation between PROP taster status and fat perception, touch, and olfaction. Physiology and Behavior, 72, 427-437. 


\section{Figures:}

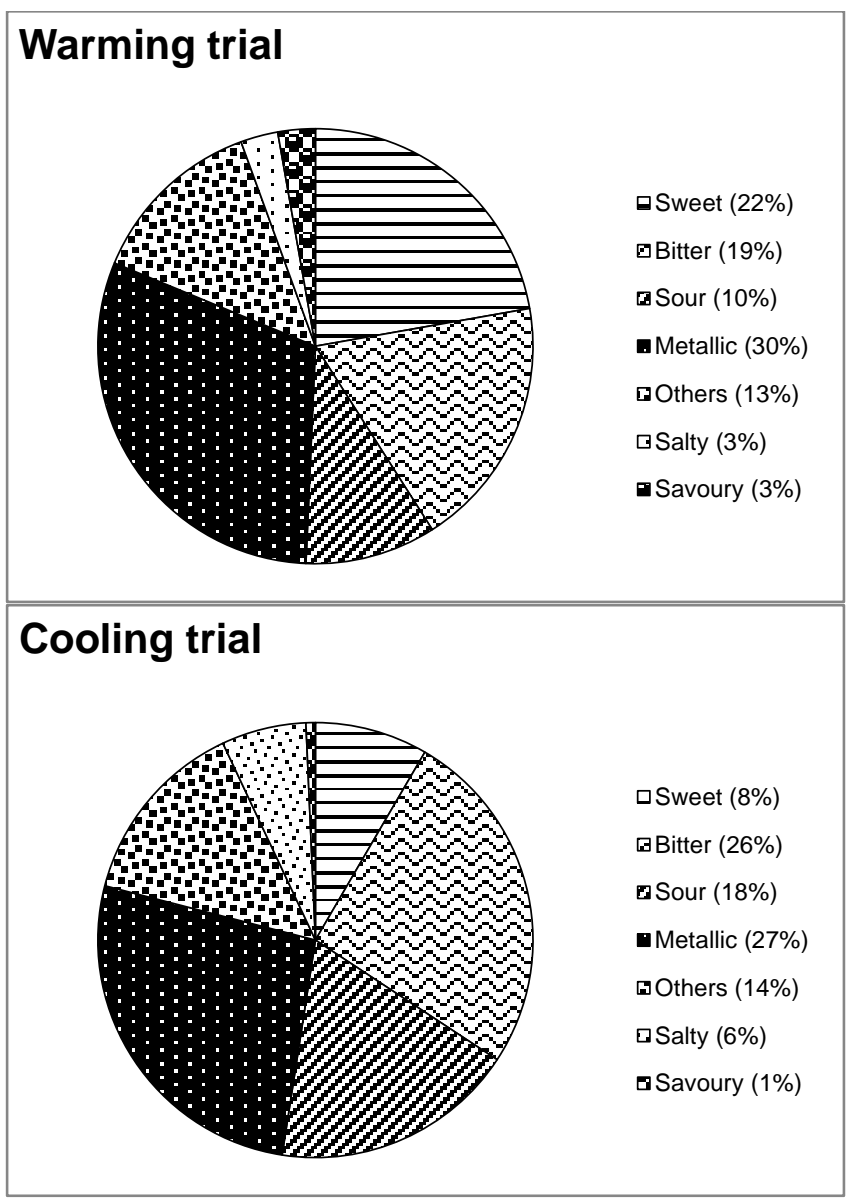

Fig.1. Percentage taste quality reported during both warming and cooling trials on the tip of the tongue by thermal tasters (TTs).

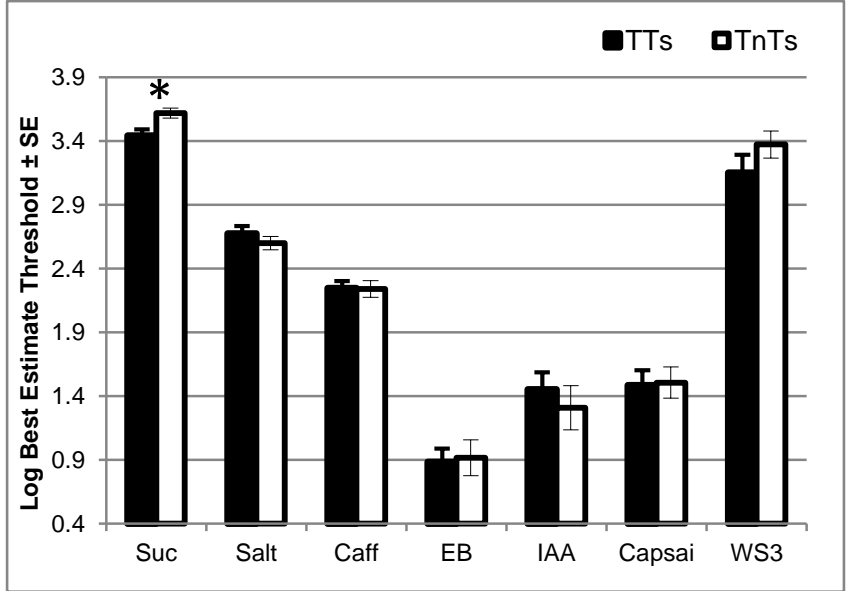

Fig.2. Effect of Thermal taster status (TTS) on detection threshold level oronasal sensations. Bars represent log best estimate threshold (BET) \pm Standard Error (SE). * $p<0.05$. (SucSucrose; Salt-Sodium Chloride; Caff- Caffeine; EB-Ethyl Butyrate; IAA-Isoamyl Acetate; Capsai-Capsaicin;WS3- N-Ethyl-2-isopropyl-5-methylcyclohexanecarboxamide). 


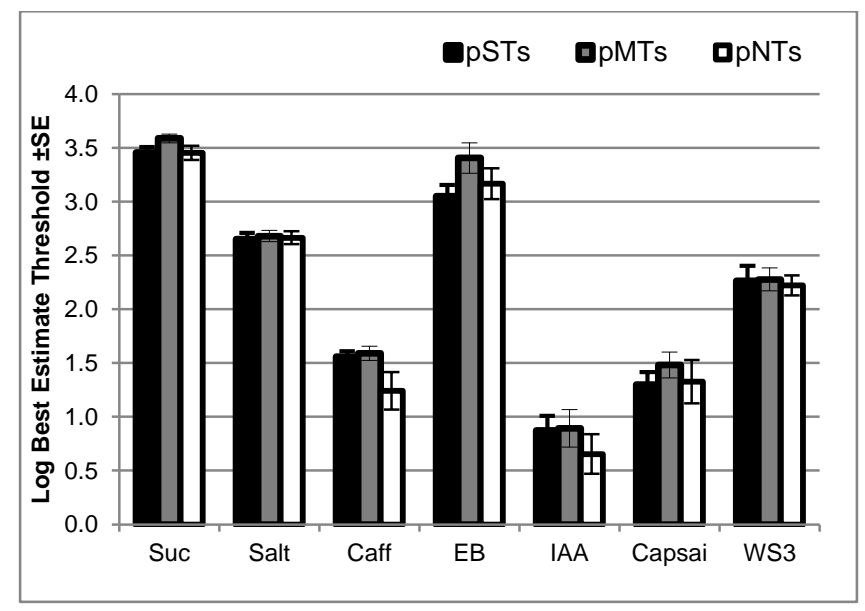

Fig. 3. Effect of PROP taster status (PTS) on detection threshold level oronasal sensations. Bars represent log best estimate threshold $(B E T) \pm$ Standard Error (SE). (Suc-Sucrose; SaltSodium Chloride; Caff-Caffeine; EB-Ethyl Butyrate; IAA-Isoamyl Acetate; CapsaiCapsaicin;WS3- N-Ethyl-2-isopropyl-5-methylcyclohexanecarboxamide).

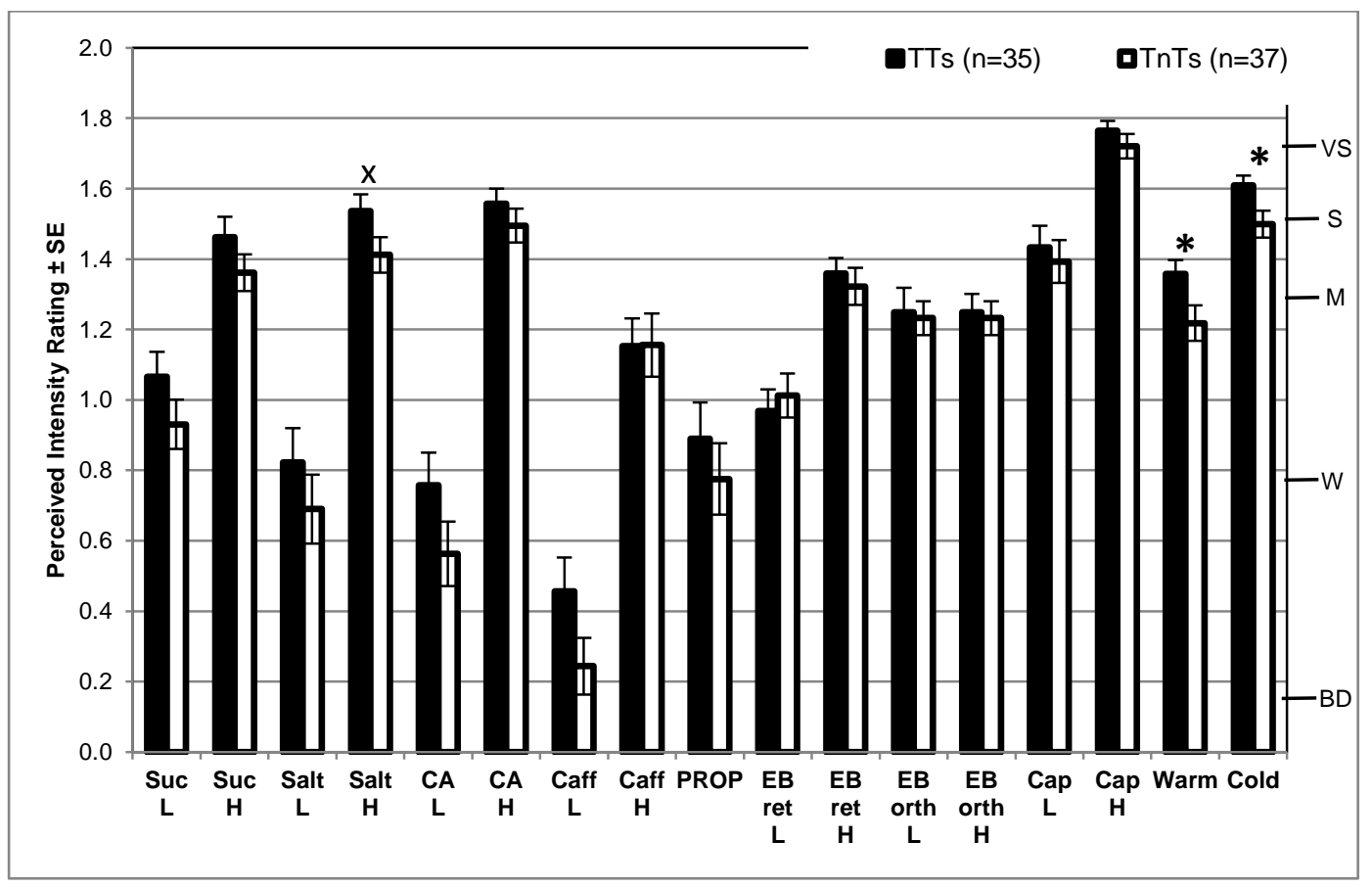

Fig. 4. Effect of TTS on supra-threshold level oronasal sensations. Bars represent log mean intensity \pm SE. ${ }^{*} p<=0.05$. ${ }^{x} p<0.1$. (BD-barely detectable, W- weak, M- moderate, S- Strong, VS-very strong on gLMS scale).(L- Low; H-High; ret- retronasal; ortho - orthonasal; SucSucrose; Salt-Sodium Chloride; CA-Citric acid; Caff-Caffeine; EB-Ethyl Butyrate; CapCapsaicin). 


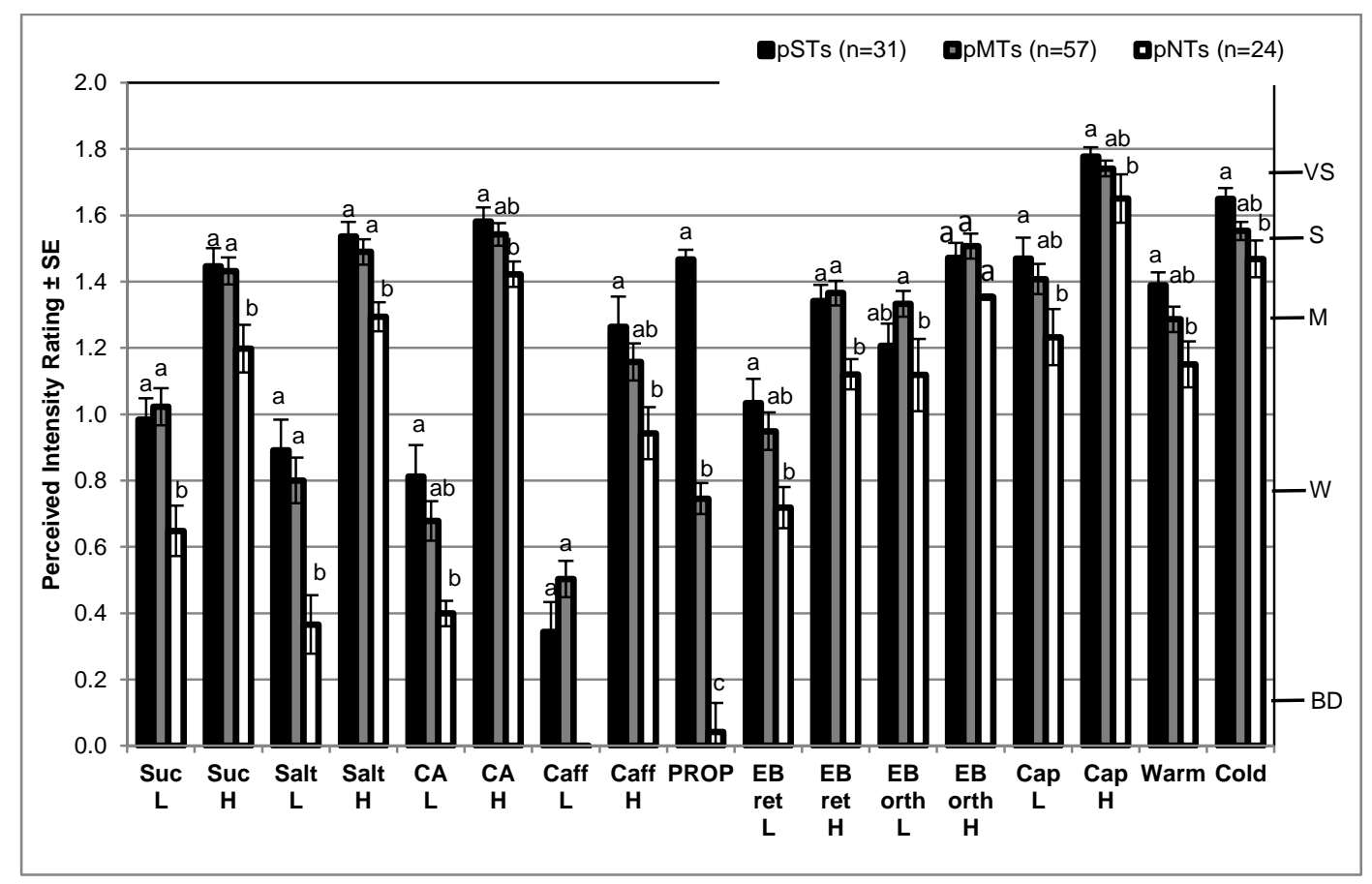

Fig. 5. Effect of PTS on supra-threshold level oronasal sensations. Bars represent log mean intensity $\pm S E$. Means with different letters within a stimulus differed at $p<0.05$. (BD-barely detectable, W- weak, M- moderate, S- Strong, VS-very strong on gLMS scale). ).(L- Low; HHigh; ret-retronasal; ortho-orthonasal; Suc-Sucrose; Salt-Sodium Chloride; CA-Citric acid; Caff-Caffeine; EB-Ethyl Butyrate; Cap-Capsaicin). 

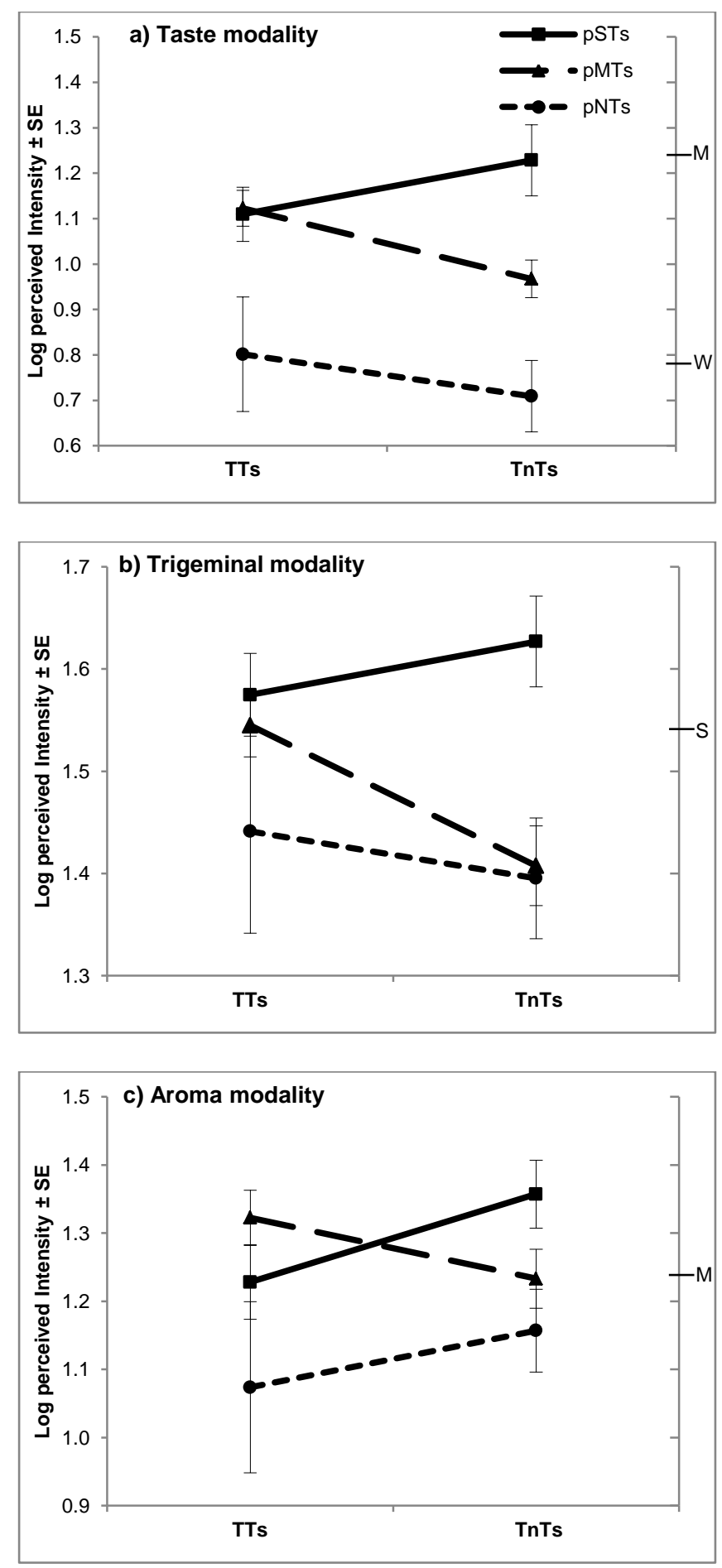

Fig. 6. TTS*PTS Interaction plot for supra-threshold ratings on each modality. a) taste modality, b) trigeminal modality, c) aroma modality. Data represent log mean intensity $\pm S E$. (W- weak, M- moderate, S- Strong on gLMS scale). 


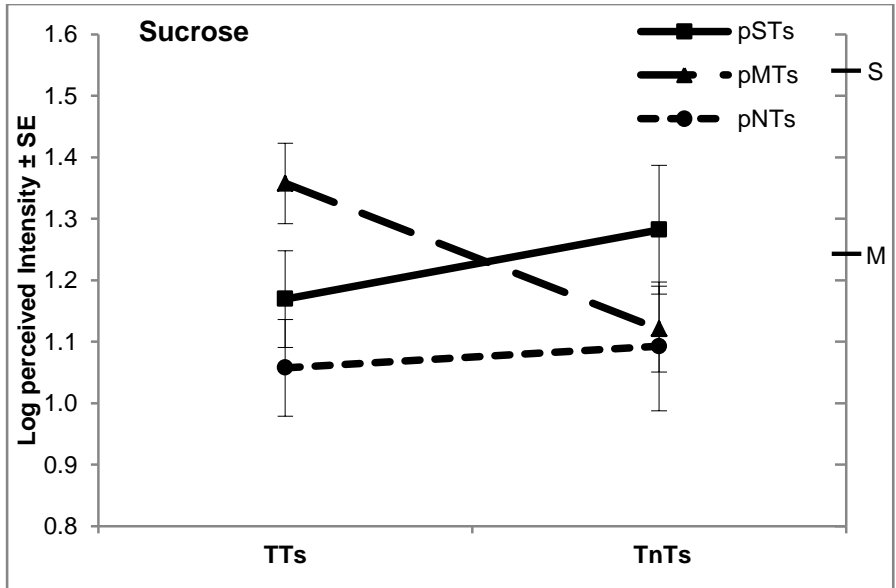

Fig. 7. Interaction plot of TTS and PTS on supra-threshold ratings on sweet attribute (sucrose). Data represent log mean intensity \pm SE. (M- moderate, S- Strong on gLMS scale). 

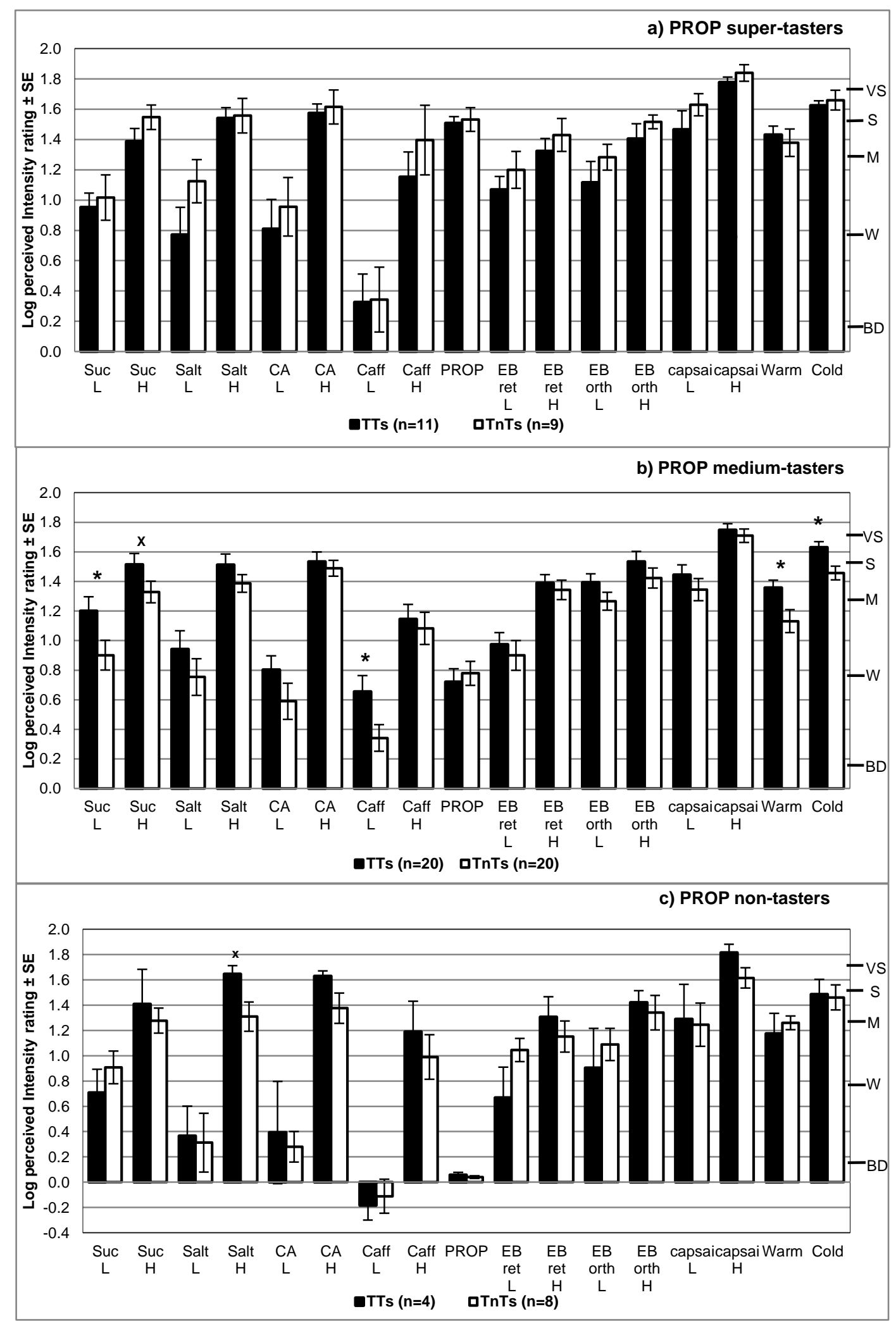

Fig. 8. Effect of TTS on supra-threshold intensity ratings for each PTS group (super-tasters, medium-tasters and non-tasters). Data represent log mean intensity \pm SE. * indicates $\mathrm{p}<0.05,{ }^{\mathrm{x}} \mathrm{p}<0.1$. (BD-barely detectable, $\mathrm{W}$ - weak, M- moderate, S- Strong, VS-very strong on gLMS scale). ).(L-Low; H-High; ret-retronasal; ortho-orthonasal; Suc-Sucrose; Salt-Sodium Chloride; CA- Citric acid; Caff-Caffeine; EB-Ethyl Butyrate; Cap-Capsaicin). 